\title{
Can Total Body Resistance Measured Using Bioelectrical Impedance Analysis Be the Index of Dehydration in Older Japanese Patients?
}

\author{
Miyuki Shimizu $^{\mathrm{a}, \mathrm{c}}$, Kensuke Kinoshita ${ }^{\mathrm{b}}$, Takami Maeno ${ }^{\mathrm{a}}$, Hiroyuki Kobayashi ${ }^{\mathrm{b}}$, \\ Tetsuhiro Maeno ${ }^{\mathrm{a}}$
}

\begin{abstract}
Background: Dehydration in older patients has long been considered a significant health problem because it implies increased morbidity and mortality. However, dehydration is detected by a combination of physical signs and blood tests. For older people dwelling at home and in nursing homes, a simple and non-invasive method for detecting dehydration by caregivers is needed. The total body resistance is measured using bioelectrical impedance analysis and is known as an indicator of dehydration. There are no data from older Japanese patients on this issue. We performed this study to examine the relationship between dehydration and total body resistance in Japan.
\end{abstract}

Methods: We performed blood tests and measured bioelectrical impedance in older outpatients aged $\geq 65$ years from the Internal Medicine Department at Mito Kyodo General Hospital. Patients were classified as dehydrated and non-dehydrated using the dehydration index with a blood urea nitrogen/creatinine ratio $>20$, and the mean total body resistance was compared between the two groups.

Results: Eighty-one patients were recruited in the study. In the dehydrated group, the mean total body resistance was $439 \Omega$ at $50 \mathrm{kHz}$, which was significantly higher than that in the non-dehydrated group (408 $\Omega, \mathrm{P}=0.038)$.

Conclusions: The total body resistance measurements can be used for simple assessment of dehydration among older Japanese patients.

Keywords: Dehydration; Total body resistance; Bioelectrical imped-

Manuscript submitted August 20, 2017, accepted September 20, 2017

aDepartment of Primary Care and Medical Education, University of Tsukuba, Tsukuba, Ibaraki, Japan

bDepartment of General Medicine, Mito Kyodo General Hospital, Mito, Ibaraki, Japan

${ }^{\mathrm{c} C o r r e s p o n d i n g ~ A u t h o r: ~ M i y u k i ~ S h i m i z u, ~ D e p a r t m e n t ~ o f ~ P r i m a r y ~ C a r e ~ a n d ~}$ Medical Education, University of Tsukuba, 1-1-1 Tennoudai, Tsukuba, Ibaraki, Japan. Email: miyuki_shimizu@medlabpart.com

doi: https://doi.org/10.14740/jocmr3170w ance analysis

\section{Introduction}

Dehydration among older patients is a significant health problem because it implies increased morbidity and mortality [1]. Dehydration exposes older patients to the risks of delirium, renal failure, falls, pressure ulcers, and constipation [1,2]. Patients are diagnosed with dehydration based on the presence of appropriate physical signs and blood test results. Blood urea nitrogen/creatinine (BUN/CRE) ratio of $>20$ is usually used as an index of dehydration.

Blood tests are performed according to the physician's order. However, a non-invasive method for detecting dehydration at home and nursing homes is needed. Common physical signs of dehydration are dry axillae [2], dry mouth, sunken eyes, decreased skin turgor, and delayed capillary refill time [3]. Although the sensitivity of these physical signs is $34-85 \%$ and the specificity is $73-95 \%$, this information is inadequate for diagnosing dehydration [3].

Bioelectrical impedance analysis (BIA) is widely used for estimating body composition in a non-invasive manner. BIA applications involve multifrequency measurements for evaluating differences in body composition caused by clinical and nutritional status. Measurements are conducted under the assumption that total body resistance changes depending on the water content of the body. Allison et al indicated that the total body resistance increased with dehydration in the United States [4]. Stooky showed the relationship between plasma tonicity and total body resistance in older American adults [5]. Furthermore, Allison reported that a total body resistance at $50 \mathrm{kHz}$ is considered an index for dehydration [4]. BIA is non-invasive and easy to perform because it does not require blood collection for detecting dehydration. However, previous studies were conducted in the United States. Although the physical built of Japanese individuals is different from that of Americans, the usefulness of total body resistance for detecting dehydration in older Japanese patients remains unclear.

In this study, the relationship between total body resistance at $50 \mathrm{kHz}$ and dehydration, classified using the BUN/ CRE ratio, was examined among older Japanese patients. 


\section{Materials and Methods}

\section{Patients}

We consecutively recruited outpatients aged $\geq 65$ years from the Internal Medicine Department at Mito Kyodo General Hospital from June to September 2016; this is a 300-bed hospital in a city with a population of approximately 300,000 . Older people who could walk to the hospital were included in the study. Patients who did not undergo blood testing as part of their medical care process with a fever of $>38^{\circ} \mathrm{C}$, or used a cardiac pacemaker were excluded from the study. Patient information including age, sex, and primary complaint was collected from data in medical records.

Written informed consent was obtained from patients by the research staff and physicians. Alternatively, written consent was sought from designated representatives if a patient was unable to provide consent because of altered levels of consciousness or cognitive dysfunction, as assessed by physicians.

\section{Blood tests}

In the initial evaluation, patients' blood samples were examined for concentrations of total protein (TP), albumin (ALB), BUN, CRE, sodium ( $\mathrm{Na})$, and plasma osmolality, and the $\mathrm{BUN} / \mathrm{CRE}$ ratio was calculated.

\section{BIA}

Following the patient interview, the patient was placed in the supine position and a physician who was blinded to the blood test results performed BIA using a bioelectrical impedance analyzer (MLT-550N, Toray Medical Co., Ltd, Tokyo, Japan). BIA measurements were performed according to the manufacturer's recommendation. Two pairs of electrodes were placed on the right wrist and right ankle. BIA, which lasted for $15 \mathrm{~s}$, involved measuring the impedance at multiple frequencies for calculating the rate of total body water [6].

\section{Statistical analyses}

According to the result of blood tests, subjects with a BUN/CRE ratio $>20$ were classified as dehydrated and the remaining were classified as non-dehydrated. Thus, we compared total body water content and resistance at $50 \mathrm{kHz}$ between the two groups.

All statistical analyses were performed using Student's unpaired $t$-test and Chi-square test using IBM SPSS version 18.0 (Japan IBM, Tokyo, Japan). A two-tailed P-value of < 0.05 was considered to be significant.

\section{Ethical consideration}

The Mito Kyodo General Hospital ethics committee approved
Table 1. Patient Characteristics

\begin{tabular}{ll}
\hline & Mean \pm SD $(\mathbf{N}=\mathbf{8 1})$ \\
\hline Male, $\mathrm{n}(\%)$ & $48(59.3)$ \\
Female, $\mathrm{n}(\%)$ & $33(40.7)$ \\
Age (years) & $75.2 \pm 7.5$ \\
Height $(\mathrm{cm})$ & $158.6 \pm 9.5$ \\
Weight $(\mathrm{kg})$ & $57.2 \pm 11.3$ \\
Total body resistance (ohms) & $419.6 \pm 66.0$ \\
TP $(\mathrm{g} / \mathrm{dL})$ & $7.1 \pm 0.6$ \\
ALB (g/dL) & $4.1 \pm 0.4$ \\
Na (mEq/L) & $140.9 \pm 5.0$ \\
BUN/CRE & $19.4 \pm 5.9$ \\
Osmolality (mOsm) & $289.6 \pm 8.0$ \\
\hline
\end{tabular}

TP: total protein; ALB: albumin; Na: sodium; BUN: blood urea nitrogen; CRE: creatinine; SD: standard deviation.

the study protocol.

\section{Results}

Eighty-one patients were recruited during the study period (Table 1). The most common reasons for seeking hospital care were digestive problems, hypertension, cold, and fatigue. The average age of the participants was 75.2 years. The mean TP and ALB concentrations were 7.1 and $4.1 \mathrm{~g} / \mathrm{dL}$, respectively. The mean Na concentration was $140.9 \mathrm{mEq} / \mathrm{L}$, mean BUN/ CRE ratio was 19.4, mean osmolality was $289.6 \mathrm{mOsm}$, and the mean total body resistance was $419.6 \Omega$ (Table 1 ).

In the dehydrated and non-dehydrated groups, the mean age was 75.1 and 75.3 years, respectively. Protein, ALB, and Na concentrations and plasma osmolality were not significantly different between the two groups. The total body resistance in the dehydrated group was greater than that in the non-dehydrated group. The mean total body resistance was 438.9 and $407.7 \Omega$ in the dehydrated and non-dehydrated groups, respectively $(\mathrm{P}=0.038)$ (Table 2$)$.

\section{Discussion}

The total body resistance among patients in the dehydrated group was greater than that in the non-dehydrated group. Notably, there is no gold standard for determining the level of dehydration in these patients. BIA is a method through which water distribution in the body can be observed in a non-invasive manner. Allison et al performed BIA to measure the volume of total body water and indicated that the total body resistance at $50 \mathrm{kHz}$ was $>550 \Omega$, a $3 \%$ decrease in body hydration [4]. The mean total body resistance in the dehydrated group was $551.8 \Omega$, which was higher than that in the normal group $(513.8 \Omega)$ [5]. In our study as well as in our previous report, the mean total body resistance of the dehydrated group was higher than that of the non-dehydrated group; however, 
Table 2. Comparison of Dehydrated and Non-Dehydrated Groups

\begin{tabular}{|c|c|c|c|}
\hline & Non-dehydrated $(\mathrm{N}=\mathbf{5 0})$ & Dehydrated $(\mathrm{N}=\mathbf{3 1})$ & $\mathbf{P}$ \\
\hline Male, n (\%) & $34(68.0)$ & $14(45.2)$ & $0.042^{\mathrm{b}}$ \\
\hline Female, n (\%) & $16(34.0)$ & $17(54.8)$ & \\
\hline Age (years) & $75.3 \pm 6.5$ & $75.1 \pm 9.1$ & $0.883^{\mathrm{a}}$ \\
\hline Height $(\mathrm{cm})$ & $159.6 \pm 9.2$ & $156.9 \pm 9.9$ & $0.212^{\mathrm{a}}$ \\
\hline Weight (kg) & $58.4 \pm 11.4$ & $55.2 \pm 10.9$ & $0.212^{\mathrm{a}}$ \\
\hline Total body resistance (ohms) & $407.7 \pm 56.8$ & $438.9 \pm 75.6$ & $0.038^{\mathrm{a}}$ \\
\hline $\mathrm{TP}(\mathrm{g} / \mathrm{dL})$ & $7.1 \pm 0.6$ & $7.1 \pm 0.5$ & $0.926^{\mathrm{a}}$ \\
\hline $\operatorname{ALB}(\mathrm{g} / \mathrm{dL})$ & $4.1 \pm 0.4$ & $4.1 \pm 0.5$ & $0.930^{\mathrm{a}}$ \\
\hline $\mathrm{Na}(\mathrm{mEq} / \mathrm{L})$ & $140.9 \pm 4.0$ & $140.8 \pm 6.3$ & $0.943^{\mathrm{a}}$ \\
\hline Osmolality (mOsm) & $288.7 \pm 9.3$ & $291.2 \pm 4.6$ & $0.197^{\mathrm{a}}$ \\
\hline
\end{tabular}

Non-dehydrated, BUN/CRE $\leq 20$; dehydrated, BUN/CRE $>20$. ${ }^{a}$-test. ${ }^{b}$ Chi-square test.

the mean total body resistance remained $438 \Omega$, which is lower than that obtained in the previous study. It is also thought to be causally related to racial differences. The total body resistance is influenced by the muscle ratio as a counterpart of body fat percentage [7]. In general, Japanese populations might have lower body fat percentage than Americans, resulting in lower total body resistance $[8,9]$. Taking the difference in physical built into consideration, an evaluation that includes the Japanese as one of the ethnic groups studied is needed.

This study indicates that BIA can be a non-invasive evaluation of dehydration when used by caregivers in settings other than hospitals. Conventional diagnosis of dehydration using a combination of blood tests and physical signs can be performed only by physicians because blood tests are conducted only by physicians' orders in a medical setting. However, older people with a higher risk of dehydration are dwelling at home and in nursing homes. BIA has potential as a simple tool for detecting dehydration at home and nursing homes.

There are several limitations to this study. First, the study was performed in only one hospital. More investigation is needed in more hospitals with several settings. Second, although measurements were conducted for general medicine patients, there were no cases of severe dehydration. It is still unclear whether total body resistance can help in detecting critical dehydration.

Our study demonstrated that total body resistance increased in dehydrated older patients. Thus, BIA has potential to be a simple tool for detecting dehydration at home and in nursing homes.

\section{Conflict of Interest}

Authors declare that they have no conflict of interest.

\section{Grant Support}

There is no grant support for this study.

\section{References}

1. Wotton K, Crannitch K, Munt R. Prevalence, risk factors and strategies to prevent dehydration in older adults. Contemp Nurse. 2008;31(1):44-56.

2. Eaton D, Bannister P, Mulley GP, Connolly MJ. Axillary sweating in clinical assessment of dehydration in ill elderly patients. BMJ. 1994;308(6939):1271.

3. McGee S, Abernethy WB, 3rd, Simel DL. The rational clinical examination. Is this patient hypovolemic? JAMA. 1999;281(11):1022-1029.

4. Allison RD, Ray Lewis A, Liedtke R, Buchmeyer ND, Frank H. Early identification of hypovolemia using total body resistance measurements in long-term care facility residents. Gend Med. 2005;2(1):19-34.

5. Stookey JD. High prevalence of plasma hypertonicity among community-dwelling older adults: results from NHANES III. J Am Diet Assoc. 2005;105(8):1231-1239.

6. Kafri MW, Myint PK, Doherty D, Wilson AH, Potter JF, Hooper L. The diagnostic accuracy of multi-frequency bioelectrical impedance analysis in diagnosing dehydration after stroke. Med Sci Monit. 2013;19:548-570.

7. Yamada Y, Masuo Y, Yokoyama K, Hashii Y, Ando S, Okayama Y, Morimoto T, et al. Proximal electrode placement improves the estimation of body composition in obese and lean elderly during segmental bioelectrical impedance analysis. Eur J Appl Physiol. 2009;107(2):135144.

8. Borrud LG, Flegal KM, Looker AC, Everhart JE, Harris TB, Shepherd JA. Body composition data for individuals 8 years of age and older: U.S. population, 1999-2004. Vital Health Stat 11. 2010;250:1-87.

9. Seino S, Shinkai S, Iijima K, Obuchi S, Fujiwara Y, Yoshida $\mathrm{H}$, Kawai $\mathrm{H}$, et al. Reference values and age differences in body composition of community-dwelling older Japanese men and women: a pooled analysis of four cohort studies. PLoS One. 2015;10(7):e0131975. 